\title{
Sirtinol, a class III HDAC inhibitor, induces apoptotic and autophagic cell death in MCF-7 human breast cancer cells
}

\author{
JING WANG ${ }^{1}$, TAE HYUNG KIM ${ }^{1}$, MEE YOUNG AHN ${ }^{1}$, JAEWON LEE ${ }^{1}$, JEE H. JUNG ${ }^{1}$, WAHN SOO CHOI ${ }^{2}$, \\ BYUNG MU LEE ${ }^{3}$, KYUING SIL YOON $^{4}$, SUNGPIL YOON ${ }^{4}$ and HYUNG SIK KIM ${ }^{1}$ \\ ${ }^{1}$ College of Pharmacy, Pusan National University, Busan 609-735; ${ }^{2}$ Department of Immunology, College of Medicine, \\ Konkuk University, Chungju-Si 380-701; ${ }^{3}$ Division of Toxicology, School of Pharmacy, Sungkyunkwan University, \\ Suwon, Gyeonggi-do; ${ }^{4}$ Research Institute, National Cancer Center, Goyang-si, Gyeonggi-do, Republic of Korea
}

Received March 19, 2012; Accepted May 25, 2012

DOI: 10.3892/ijo.2012.1534

\begin{abstract}
Sirtuins (SIRTs), NAD ${ }^{+}$-dependent class III histone deacetylases (HDACs), play an important role in the regulation of cell division, survival and senescence. Although a number of effective SIRT inhibitors have been developed, little is known about the specific mechanisms of their anticancer activity. In this study, we investigated the anticancer effects of sirtinol, a SIRT inhibitor, on MCF-7 human breast cancer cells. Apoptotic and autophagic cell death were measured. Sirtinol significantly inhibited the proliferation of MCF-7 cells in a concentration-dependent manner. The $\mathrm{IC}_{50}$ values of sirtinol were $48.6 \mu \mathrm{M}(24 \mathrm{~h})$ and $43.5 \mu \mathrm{M}(48 \mathrm{~h})$ in MCF-7 cells. As expected, sirtinol significantly increased the acetylation of p53, which has been reported to be a target of SIRT1/2. Flow cytometry analysis revealed that sirtinol significantly increased the G1 phase of the cell cycle. The upregulation of Bax, downregulation of Bcl-2 and cytochrome $c$ release into the cytoplasm, which are considered as mechanisms of apoptotic cell death, were observed in the MCF-7 cells treated with sirtinol. The Annexin V-FITC assay was used to confirm sirtinol-induced apoptotic cell death. Furthermore, the expression of LC3-II, an autophagy-related molecule, was significantly increased in MCF-7 cells after sirtinol treatment. Autophagic cell death was confirmed by acridine orange and monodansylcadaverine (MDC) staining. Of note, pre-treatment with 3-methyladenine (3-MA) increased the sirtinol-induced MCF-7 cell cytotoxicity, which is associated with blocking autophagic cell death and increasing apoptotic cell death. Based on our results, the downregulation of SIRT1/2 expression may play an important role in the regulation of breast cancer cell death; thus, SIRT1/2 may be a novel molecular target for cancer therapy and these
\end{abstract}

Correspondence to: Professor Hyung Sik Kim, College of Pharmacy, Pusan National University, San 30, Jangjeon-dong, Geumjeung-gu, Busan 609-735, Republic of Korea

E-mail: hkims@pusan.ac.kr

Key words: sirtinol, histone deacetylase inhibitor, sirtuin, breast cancer, apoptosis, autophagy findings may provide a molecular basis for targeting SIRT1/2 in future cancer therapy.

\section{Introduction}

Breast cancer is the most frequently diagnosed cancer in women and is one of the leading causes of cancer mortality worldwide (1). Approximately 4,000 new cases of female breast cancer are diagnosed and approximately 1,000 women succumb to the disease each year in Korea (2). Candidate molecular pathways of cancer therapy emphasize signaling networks that control cell proliferation or survival $(3,4)$. However, the involvement of signaling networks in breast cancer therapy is not clearly understood. There is an urgent need to develop new strategies for breast cancer therapy.

Recently, certain studies demonstrated that autophagic cell death plays an important role in regulating cell death in breast cancer cells with acquired-resistance to various treatments (5-7). Autophagy is a catabolic pathway whereby cytoplasmic proteins and organelles are sequestered in vacuoles and delivered to lysosomes for degradation and recycling (8). The induction of autophagy has also been observed in malignant cells following treatment with histone deacetylase (HDAC) inhibitors. HDACs comprise a superfamily of proteins involved in a wide range of cellular functions, including regulation of transcription, cell proliferation and cell death $(9,10)$. HDACs are divided into four classes (I-IV) and regulate the expression and activity of numerous proteins involved in cancer. Sirtuins (also known as SIRTs) are $\mathrm{NAD}^{+}$-dependent class III HDACs (11). In mammals, seven SIRT homologues have been identified that primarily possess HDACs (SIRT1, SIRT2, SIRT3 and SIRT5) or monoribosyltransferase activity (SIRT4 and SIRT6), which target histone and various non-histone proteins in distinct subcellular locations $(12,13)$.

As SIRT1 blocks senescence, cell differentiation and stressinduced apoptosis, and promotes cell growth, angiogenesis and vasodilation (14), SIRT1 overexpression can enhance tumor growth and promote cell survival in response to stress and drug resistance; moreover, SIRT1 is upregulated in a spectrum of cancers $(15,16)$. SIRT1 can induce chromatin silencing through the deacetylation of histones H1, H3 and H4 (17) and can modulate cell survival by regulating the $\mathrm{Ku}$ autoantigen, $\mathrm{Ku} 70$ (18), 
NF- $\mathrm{BB}(19)$, FOXO proteins $(20,21)$ and p300 (22). The putative role of SIRT1 in cancer biology was first postulated when p53 was identified as a direct substrate (23). Acetylated Lys382 within p53 has been identified as a direct target of SIRT1, whereby p53 deacetylation has been shown to induce apoptosis. Therefore, SIRT1 has been implicated in the initiation and progression of various malignancies.

Conversely, the specific SIRT1 inhibitor, sirtinol, does not inhibit class I and II HDACs $(24,25)$. The first known SIRT inhibitors can be classified into two groups: substances that inhibit $\mathrm{NAD}^{+}$-dependent reactions in general, such as nicotinamide $(26,27)$ and SIRT-specific inhibitors, such as spitomicin (24), sirtinol (28), cambinol (29), dihydrocoumarin (30) and certain indoles (31). Their common feature is that they have antitumor properties; however, their molecular mechanisms of action vary and are not yet fully understood.

In the present study, we focused on the anticancer effect of sirtinol, a molecule which has a potent inhibitory effect on SIRT1. We also focused on the effect of sirtinol on the tumor suppressor, p53, and its acetylation status. The role of acetylation as a protein post-translational modification, independent of histone modification, may also play a critical role in cell fate and thus, tumorigenesis. Additionally, the anticancer effects of sirtinol on cell viability, cell cycle regulation and modulation of apoptosis- and autophagy-related molecules were investigated.

\section{Materials and methods}

Reagents. Sirtinol was purchased from Sigma-Aldrich (St. Louis, MO, USA). Culture medium and its supplements including antibiotics and fetal bovine serum (FBS) were purchased from Gibco Invitrogen Corp. (Carlsbad, CA, USA). Primary antibodies against Atg5, Atg7, $\beta$-actin, beclin-1, cleaved caspase 7, caspase 7, cleaved caspase 9, Cdc2, cyclin A, cyclin B1, cyclin D1, cyclin E, cytochrome $c$, HDACs, LC3B and $\mathrm{p} 21$, and horseradish peroxidase-conjugated secondary antibody were purchased from Cell Signaling Technology, Inc. (Danvers, MA, USA). Bax, Bcl-2, Cdk4, Cdk6, Cdk2, histone H1, poly(ADP-ribose) polymerase (PARP), p53, Ac-p53, and p27 were purchased from Santa Cruz Biotechnology (Santa Cruz, CA, USA); The Annexin V-FITC apoptosis detection kit I was from BD Biosciences (San Diego, CA, USA). All other chemicals were purchased from Sigma-Aldrich. Sirtinol was dissolved in dimethyl sulfoxide (DMSO) and stored at $-20^{\circ} \mathrm{C}$ until use. Sirtinol was diluted to the appropriate concentrations in culture medium containing $1 \%$ FBS. The final concentration of DMSO was $<0.1 \%(\mathrm{vol} / \mathrm{vol})$, and was also present in the corresponding control.

Cell lines and culture medium. MCF-7 human breast cancer cells were purchased from the American Type Culture Collection (Manassas, VA, USA). The cells were grown in Dulbecco's modified Eagle's medium (Gibco, Rockville, MD, USA) containing $10 \%$ heat-inactivated FBS. Cells were maintained as monolayers in a humidified atmosphere containing $5 \% \mathrm{CO}_{2}$ at $37^{\circ} \mathrm{C}$ and the culture medium was replaced every two days. After $48 \mathrm{~h}$ of incubation, the culture medium was replaced with treatment medium containing the desired concentrations of chemicals.
Cell viability assay. Cell viability was determined using 3-(4,5-dimethylthiazol-2-yl)-2,5-diphenyltetrazolium bromide (MTT, $5 \mathrm{mg} / \mathrm{ml}$, Sigma). The cultures were initiated in 96-well plates at a density of $2.5 \times 10^{3}$ cells per well. After $48 \mathrm{~h}$ of incubation, the cells were treated with various concentrations of sirtinol and cultured for 24 or $48 \mathrm{~h}$. At the end of the treatment period, $100 \mu \mathrm{l}$ of 1X MTT reagent were added to each well and incubated for $4 \mathrm{~h}$ at $37^{\circ} \mathrm{C}$ in the dark. After incubation, the supernatant was aspirated and formazan crystals were dissolved in $100 \mu \mathrm{l}$ of DMSO at $37^{\circ} \mathrm{C}$ for 15 min with gentle agitation. The absorbance per well was measured at $540 \mathrm{~nm}$ using the VersaMax Microplate reader (Molecular Devices Corp., CA, USA). Data from three independent experiments were analyzed and then normalized to the absorbance of wells containing medium only ( $0 \%)$ and untreated cells $(100 \%)$. $\mathrm{IC}_{50}$ values were calculated from sigmoidal dose-response curves using SigmaPlot 10.0 software.

Protein extraction and western blot analysis. MCF-7 cells were treated with sirtinol $(2,10$, or $50 \mu \mathrm{M})$ for $48 \mathrm{~h}$. Cells were harvested by trypsinization and washed twice with cold phosphate-buffered saline (PBS). For total protein isolation, cells were suspended in PRO-PREP ${ }^{\mathrm{TM}}$ protein extract solution (Intron, Seongnam, Korea) and protein concentrations were measured using the protein assay kit (Bio-Rad, Hercules, CA, USA) according to the manufacturer's instructions. Equivalent amounts of proteins were resolved and electrophoresed using SDS-PAGE on a $6-15 \%$ gel. Gels were transferred to polyvinylidene difluoride (PVDF) membranes (Millipore, Billerica, MA, USA) and membranes were blocked with blocking buffer (TNA buffer containing 5\% skim milk) for $1 \mathrm{~h}$. Subsequently, the membranes were incubated with primary antibodies at $4^{\circ} \mathrm{C}$ overnight. After washing for $1 \mathrm{~h}$ with TNA buffer (10 mM Tris-Cl, pH 7.6, $100 \mathrm{mM} \mathrm{NaCl}$ and $0.5 \%$ Tween-20), the membranes were incubated with horseradish peroxidaseconjugated anti-mouse or anti-rabbit antibody $(1: 10,000$, Santa Cruz Biotechnology) for $30 \mathrm{~min}$ at room temperature and then washed for $1 \mathrm{~h}$ with TNA buffer. The blots were developed using an enhanced chemiluminescence (ECL)-plus kit (Amersham Biosciences, Buckinghamshire, UK).

Flow cytometry analysis. The cells were treated with various concentrations of sirtinol $(2,10$, or $50 \mu \mathrm{M})$ for $48 \mathrm{~h}$. The total number of cells, including the ones in suspension and those adhered on the walls, were harvested separately for sub-G1 or other cell cycle stages, respectively, and washed in $1 \%$ bovine serum albumin (BSA) before fixing in 95\% ice-cold ethanol containing $0.5 \%$ Tween- 20 for $1 \mathrm{~h}$ at $-20^{\circ} \mathrm{C}$. The cells $\left(1 \times 10^{6}\right)$ were washed in $1 \%$ BSA, stained with cold propidium iodide (PI) staining solution $(10 \mu \mathrm{g} / \mathrm{ml} \mathrm{PI}$ and $100 \mu \mathrm{g} / \mathrm{ml}$ RNase in PBS) and incubated in the dark for $30 \mathrm{~min}$ at room temperature. Data acquisition and analysis were carried out using a flow cytometry system (Becton-Dickinson, San Jose, CA, USA).

4',6-Diamidino-2-phenylindole (DAPI) staining. Morphological changes in the nuclear chromatin of apoptotic cells were identified by staining with the DNA binding dye DAPI. Cells were grown in 6-well plates at a density of $1 \times 10^{5}$ cells per well followed by the desired treatment. After $48 \mathrm{~h}$ of incubation, the cells were washed with cold PBS, fixed with methanol 

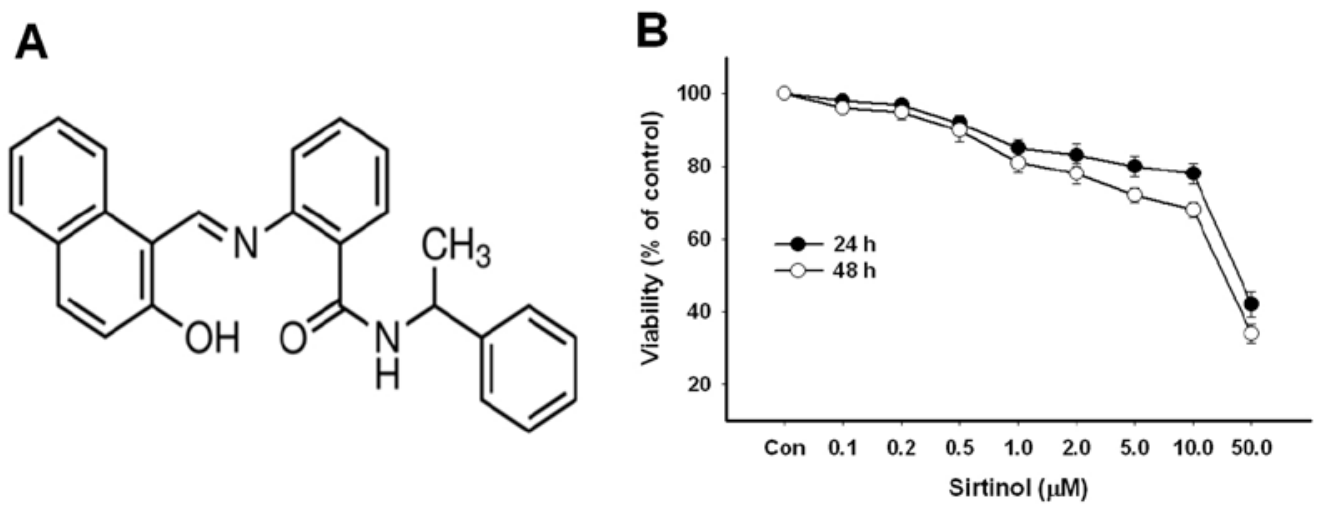

Figure 1. Assessment of the MCF-7 cell cytotoxicity induced by sirtinol. (A) Chemical structure of sirtinol used in the present study. (B) The cells were treated with sirtinol at various concentrations $(0.1-50 \mu \mathrm{M})$ for 24 and $48 \mathrm{~h}$. Viable cells were detected by MTT assay and viability was determined as the ratio between treated cells and untreated controls. The data are represented as the means \pm SEM from three independent experiments.

for $30 \mathrm{~min}$, rewashed and stained with $200 \mu \mathrm{l}$ of DAPI solution $(1 \mu \mathrm{g} / \mathrm{ml})$ at $37^{\circ} \mathrm{C}$ for $30 \mathrm{~min}$. After the staining solution was removed, the apoptotic cells were visualized using a fluorescence confocal microscope at x400 magnification.

Annexin V-FITC binding assay. The Annexin V-FITC binding assay was performed according to manufacturer's instructions using the Annexin V-FITC detection kit I (BD Biosciences). The cells were treated with sirtinol $(2,10$, or $50 \mu \mathrm{M})$ for $48 \mathrm{~h}$. The total number of cells was counted by trypsinization and washed twice with cold PBS. The cell pellet was resuspended with $100 \mu \mathrm{l}$ of binding buffer at a density of $1 \times 10^{3}$ cells per $\mathrm{ml}$ and incubated with $5 \mu \mathrm{l}$ of FITC-conjugated Annexin V and $5 \mu \mathrm{l}$ of PI for $15 \mathrm{~min}$ at room temperature in the dark. A total of $400 \mu \mathrm{l}$ of $1 \mathrm{X}$ binding buffer was added to each sample tube and the samples were immediately analyzed by FACSCalibur (Becton-Dickinson).

Caspase activity analysis. The cultures were initiated in 6-well plates at a density of $1 \times 10^{5}$ cells per well. Cells were allowed to attach for $48 \mathrm{~h}$ and exposed to SAHA for $48 \mathrm{~h}$. Caspase 8 and caspase 9 activities in the cell lysate were measured using colorimetric assay kits (Biovision Ins., CA, USA) as described in the manufacturer's protocol. The kits used in this study utilized synthetic tetrapeptides labeled with $p$-nitroanilide (pNA). Briefly, fifty microliters $(100 \mu \mathrm{g})$ of cell lysate were incubated with $50 \mu \mathrm{l}$ of $2 \mathrm{x}$ reaction buffer and $2 \mu \mathrm{l}$ of IETD-pNA for capase 8 or LEHD-pNA for caspase 9 at $37^{\circ} \mathrm{C}$ for $2 \mathrm{~h}$. A reading was then taken from a spectrophotometer at $405 \mathrm{~nm}$ with a VERS Amax Microplate Reader (Molecular Devices Corp.). In case of caspase 8 activity, a reading was taken from a fluorescence microtiter plate reader. Caspase 7 activities in the cell lysate were measured using caspase 7 immunoassay kits (Biovision Ins., CA, USA) as described in the manufacturer's protocol. Briefly, the assay utilizes caspase 7 polyclonal antibody to capture activated caspase 7 from cell lysates. Substrate DEVD-AFC is then added that is cleaved proportionally to the amount of activated caspase 7 in the cell lysate. The cleavage generates free AFC which is then analyzed fluorometrically $(\mathrm{Ex} . / \mathrm{Em} .=400 / 505 \mathrm{~nm})$ using a fluorescence plate reader. The assay ensures absolute specific detection of caspase 7. Other known caspases and non-specific proteases are not detected.
Acridine orange staining. MCF-7 cells were seeded in T-25 flasks and at $70 \%$ confluence the cells were treated with sirtinol for $48 \mathrm{~h}$. At the appropriate time-points, cells were incubated with acridine orange $(1 \mu \mathrm{g} / \mathrm{ml})$ in serum-free medium at $37^{\circ} \mathrm{C}$ for $15 \mathrm{~min}$. The acridine orange was removed and fluorescent micrographs were obtained using an inverted fluorescence microscope (Olympus FV10i; Olympus, Tokyo, Japan). The cytoplasm and nucleus of the stained cells fluoresced bright green, whereas the acidic autophagic vacuoles fluoresced bright red. Cells were treated with $200 \mathrm{nmol} / \mathrm{l}$ bafilomycin A1 for $30 \mathrm{~min}$ before the addition of acridine orange to inhibit the acidification of autophagic vacuoles. To quantify the development of acidic vesicular organelles (AVOs), sirtinol-treated or control cells were stained with acridine orange $(1 \mu \mathrm{g} / \mathrm{ml})$ for $15 \mathrm{~min}$ and removed from the plate with trypsin-EDTA, and collected in phenol red-free growth medium. Green (510$530 \mathrm{~nm})$ and red $(650 \mathrm{~nm})$ fluorescence emission from $1 \times 10^{4}$ cells illuminated with blue $(488 \mathrm{~nm})$ excitation light were measured with a FACSCalibur.

Monodansylcadaverine (MDC) incorporation assay. Autophagic vacuoles were also detected with MDC by incubating the cells with $\mathrm{MDC}(50 \mu \mathrm{mol} / \mathrm{l})$ in $\mathrm{PBS}$ at $37^{\circ} \mathrm{C}$ for $10 \mathrm{~min}$. After incubation, the cells were washed four times with cold PBS and fixed with $3.75 \%$ paraformaldehyde in PBS. The cells were immediately analyzed by fluorescence microscopy using an inverted microscope (Olympus FV10i) equipped with a filter system (excitation wavelength, $380 \mathrm{~nm}$; emission filter, $525 \mathrm{~nm})$.

\section{Results}

Sirtinol suppresses the growth of MCF-7 breast cancer cells. We first assessed the cytotoxicity of sirtinol in MCF-7 cells by using the MTT assay. The chemical structure of sirtinol is shown in Fig. 1A. Sirtinol significantly reduced the growth of MCF-7 cells in a concentration- and time-dependent manner. The $\mathrm{IC}_{50}$ values of sirtinol were $48.6 \mu \mathrm{M}$ and $43.5 \mu \mathrm{M}$ after 24 and $48 \mathrm{~h}$ of treatment, respectively (Fig. 1B).

Sirtinol decreases SIRT protein expression in MCF-7 cells. The expression levels of SIRT1, SIRT2 and SIRT3 were 

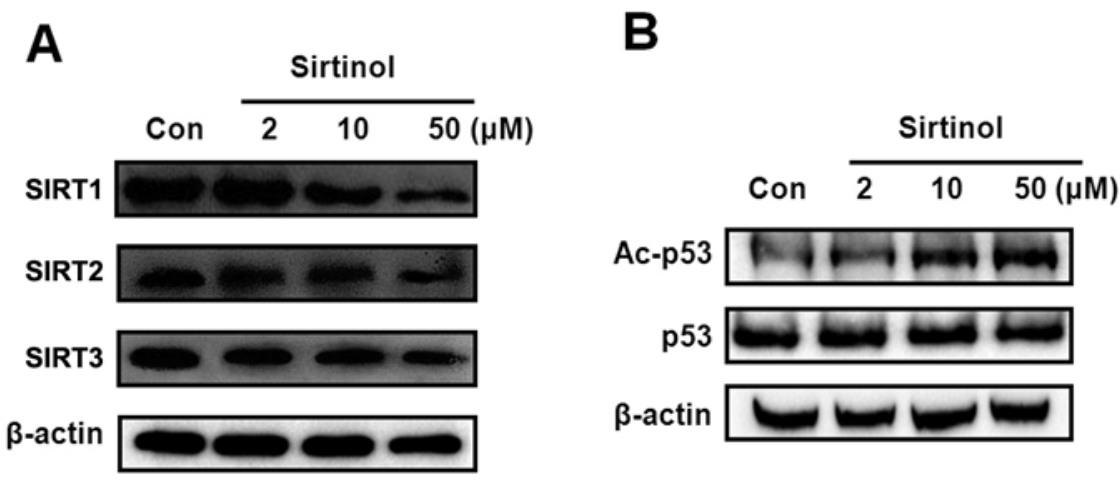

Figure 2. Effect of sirtinol on sirtuin (SIRT) and p53 expression in MCF-7 cells. MCF-7 cells were treated with sirtinol (2, 10 and $50 \mu \mathrm{M})$ for $48 \mathrm{~h}$. (A) Expression levels of SIRT1, SIRT2 and SIRT3 in treated cells were detected using western blot analysis. $\beta$-actin was used as an equal loading control. (B) Effect of sirtinol on p53 and acetylated p53 (Ac-p53) expression. MCF-7 cells were treated for $48 \mathrm{~h}$ with sirtinol $(2,10$ and $50 \mu \mathrm{M})$ then protein expression was detected by western blot analysis. Data are representative of three independent experiments. Con, control.

A
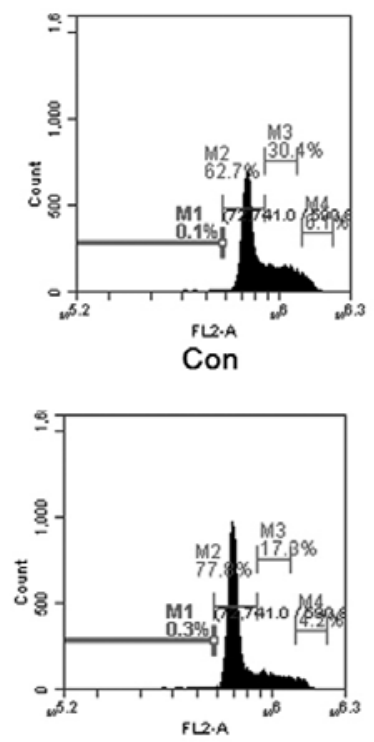

Sirtinol $(10 \mu \mathrm{M})$
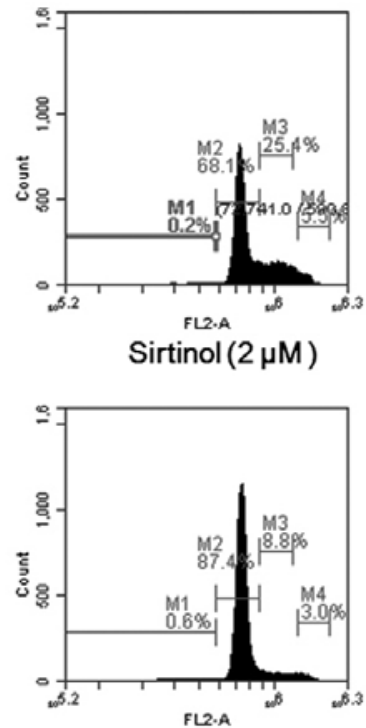

Sirtinol $(50 \mu \mathrm{M})$
B

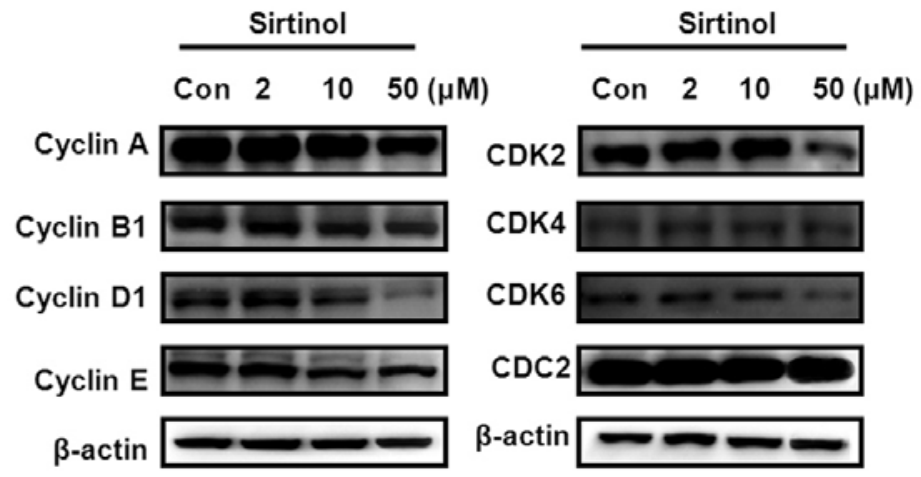

Sirtinol

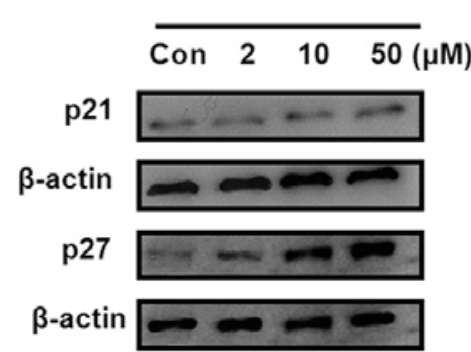

Figure 3. Effect of sirtinol on cell cycle distribution. (A) MCF-7 cells were treated with the indicated concentrations of sirtinol (2, 10 and $50 \mu \mathrm{M}$ ) for $48 \mathrm{~h}$. The cells stained with propidium iodide (PI) were subjected to flow cytometric analysis to determine the cell distributions at each phase of the cell cycle. (B) Effect of sirtinol on expression levels of cell cycle regulatory proteins. MCF-7 cells were treated with sirtinol (2, 10 and $50 \mu \mathrm{M})$ for $48 \mathrm{~h}$ and then the protein level was detected by western blot analysis. Data are representative of three independent experiments. Con, control.

measured by western blot analysis. The experiment revealed that sirtinol significantly decreased SIRT1 expression (Fig. 2A). Mammalian SIRT1 is the direct homologue of yeast SIRT2 and belongs to the $\mathrm{NAD}^{+}$-dependent HDAC family and has been implicated as a regulator of a variety of important biological processes, such as aging, metabolism and stress resistance (32). To further investigate the distinct effects of the SIRT inhibitor on cell fate, we measured the acetylated status of the SIRT1/2 target, p53. Sirtinol significantly increased the acetylated p53 level, whereas p53 acetylation was also accompanied by an induction of p53 stability (Fig. 2B).
Sirtinol affects cell cycle regulation in MCF-7 cells. To confirm that sirtinol affects cell cycle regulation in MCF-7 cells, we measured the cell cycle distribution using flow cytometry analyses. Sirtinol significantly induced G1 phase arrest at $48 \mathrm{~h}$ (Fig. 3A). The percentage of the sub-G1 population, which is indicative of apoptosis, increased after treatment with sirtinol in MCF-7 cells. We also examined the expression levels of cell cycle-regulated proteins by western blot analysis. Sirtinol significantly decreased the expression of cyclin B1, cyclin D1, CDK2 and CDK6, indicating that these molecules are closely associated with the G1 cell cycle check-point. Furthermore, 


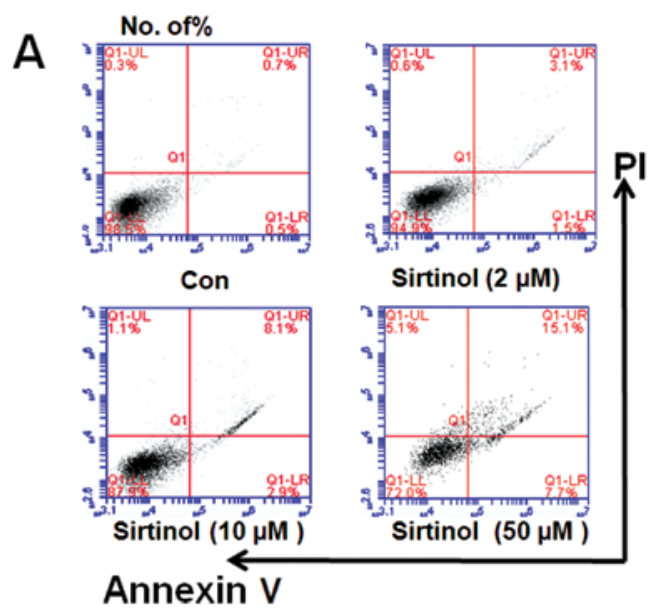

C

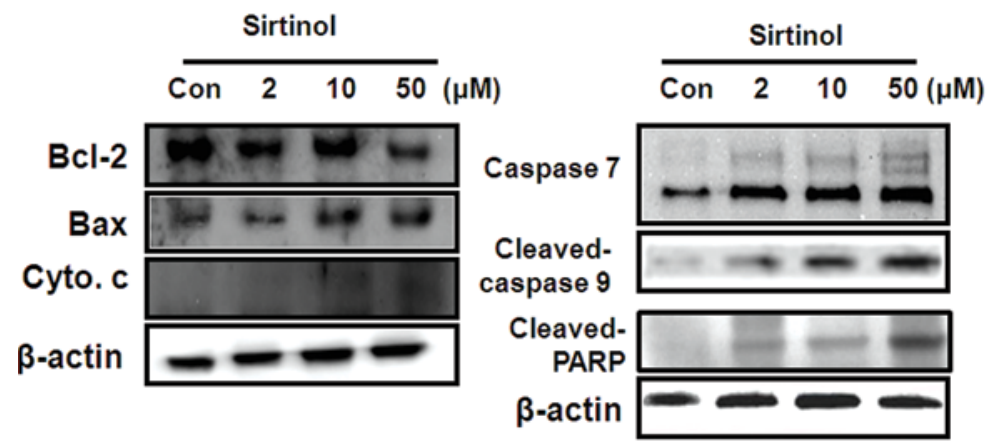

B

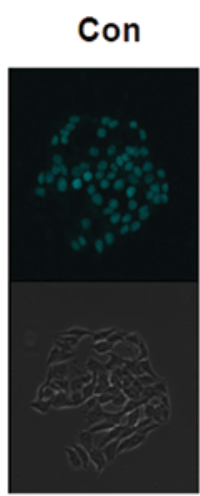

Sirtinol $(\mu \mathrm{M})$

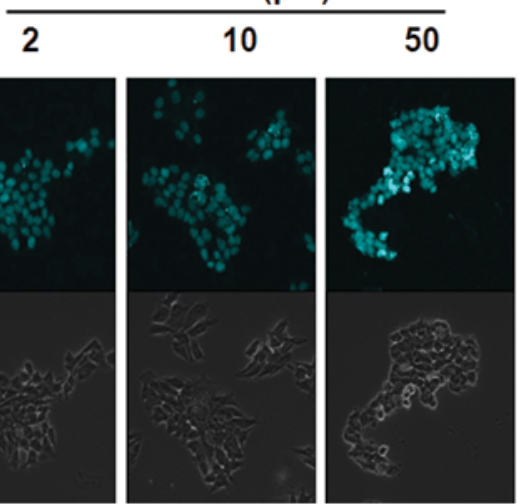

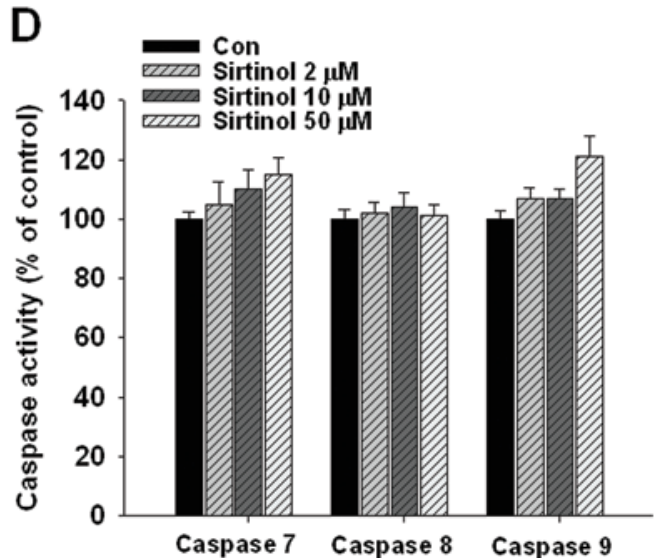

Figure 4. Effect of sirtinol on apoptosis. (A) Sirtinol-induced apoptosis in MCF-7 cells was examined by Annexin V-propidium iodide (PI) binding assay. Cells were treated with sirtinol for $48 \mathrm{~h}$ at different concentrations as indicated. Representative flow cytometry scatter plots depict percentage of Annexin $\mathrm{V}$ staining at $48 \mathrm{~h}$ after sirtinol treatment. (B) Effects of sirtinol on nuclear morphological changes in MCF-7 cells. Cells were treated with sirtinol for $48 \mathrm{~h}$ at the indicated concentrations, followed by fixation and DAPI staining. Upper images were observed in a bright field, while lower images show corresponding DAPI staining under a fluorescence microscope. Apoptotic cells are marked by arrows. These images were captured at x400. (C) Effect of sirtinol on expression of proteins related with apoptotic cell death via the mitochondrial pathway. MCF-7 cells were treated with sirtinol for $48 \mathrm{~h}$ and western blot analysis was carried out to detect the apoptosis related to protein expression. In the cell lines, a high concentration of sirtinol induced PARP cleavage, activated caspase 7 and induced the release of cytochrome $c$, while Bax expression increased and that of Bcl-2 decreased. The protein levels were normalized by comparison to the levels of $\beta$-actin. (D) Effects of sirtinol on caspase activity in MCF-7 cells. The cells were treated with the indicated concentrations of sirtinol for 48 h. The cytosolic extracts of cells were prepared and caspase 7 , caspase 8 and caspase 9 activities were measured using an assay kit. The data are presented as the means \pm SEM from three independent experiments. Con, control; Cyto. c, cytochrome $c$.

sirtinol significantly increased p21 and p27 expression in MCF-7 cells (Fig. 3B).

Sirtinol induces apoptosis in MCF-7 cells. To elucidate the mechanism underlying the cytotoxic effect of sirtinol on MCF-7 cells, apoptotic cell death was measured by Annexin V-FITC assay, DAPI staining and western blot analysis. Sirtinol (10 and $50 \mu \mathrm{M})$ significantly increased the population of late-stage apoptotic cells (Fig. 4A). DAPI staining was used to confirm the effect of sirtinol on apoptosis in MCF-7 cells. Sirtinol increased the number of apoptotic nuclei (condensed or fragmented chromatin) compared with the control culture, which showed enhanced fluorescence staining with DAPI (Fig. 4B). To determine the apoptotic pathway involved, we measured the expression of apoptosisrelated protein levels by western blot analysis. Sirtinol $(50 \mu \mathrm{M})$ significantly increased the cleavage of PARP, release of cytochrome $c$ and the expression of Bax, and decreased the expression of Bcl-2 in MCF-7 cells (Fig. 4C). The activities of caspase 7 and caspase 9 were slightly increased in MCF-7 cells treated with sirtinol; however, caspase 8 activity was not altered (Fig. 4D).

Sirtinol induces autophagy in MCF-7 cells. To evaluate autophagic cell death induced by sirtinol, western blot analysis, acridine orange and MDC staining were performed. The conversion of the soluble form of LC3-I to the autophagic vesicle-associated form, LC3-II, is considered a specific marker of autophagosome promotion. Sirtinol significantly increased the level of LC3-II and decreased the unconjugated LC3-I levels. Moreover, similar to the LC3-II expression pattern, the level of beclin-1, known as Atg6, was increased by sirtinol treatment (Fig. 5A).

The induction of autophagy was confirmed by acridine orange and MDC staining. The vital dyes, acridine orange and MDC, are commonly used to study autophagy. Acridine orange is a lysotropic dye that accumulates in acidic organelles in a $\mathrm{pH}$-dependent manner. At a neutral $\mathrm{pH}$, acridine orange 
A
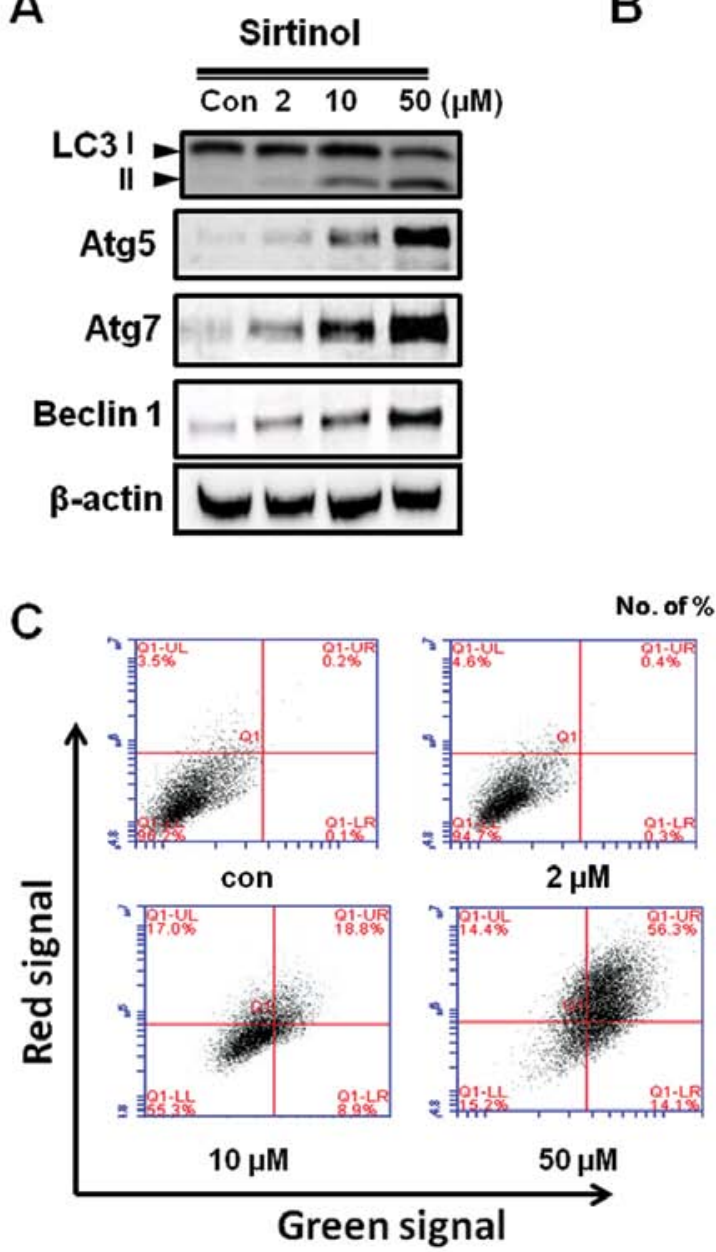

B

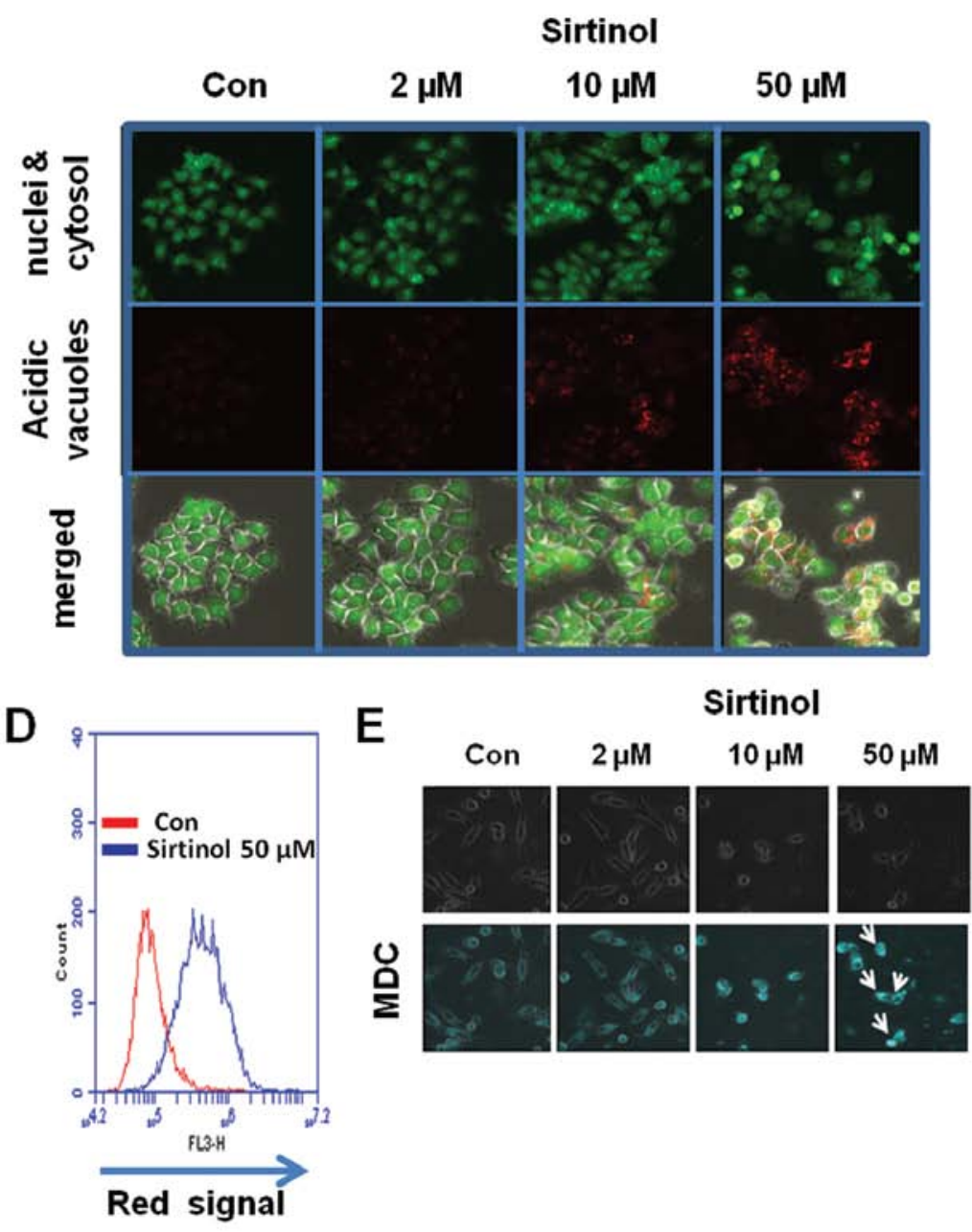

Figure 5. Effects of sirtinol on the expression of autophagy-related proteins in MCF-7 cells. (A) Western blot analysis was performed with beclin-1, LC3, Atg5 and Atg7 antibodies. Equal loading and transfer were verified by reprobing the membranes with $\beta$-actin antibody. (B) Immunofluorescence microscopy of acridine orange-stained MCF-7 cells treated for $48 \mathrm{~h}$ with the indicated drug treatment (using an Olympus confocal microscope, FV10i; magnification, x600). (C and D) Histogram profiles of control- and drug-treated cells that were read flow cytometrically. (E) Monodansylcadaverine (MDC) staining showing that autophagy was activated in MCF-7 cells after treatment with sirtinol. Cells were examined by an Olympus confocal microscope (FV10i). Magnification, x600. Con, control.

is a hydrophobic green fluorescent molecule. However, within acidic vesicles, acridine orange becomes protonated and trapped within the organelle and forms aggregates that emit bright red fluorescence (33). MDC is another popular autofluorescent marker that preferentially accumulates in autophagic vacuoles. While acridine orange staining in lysosomes is primarily due to ion trapping, MDC accumulation in auotphagic vacuoles is due to a combination of ion trapping and specific interactions with vacuole membrane lipids $(34,35)$. With acridine orange staining, control cells primarily exhibited green fluorescence with minimal red fluorescence, indicating a lack of AVOs; however, sirtinol-treated cells showed a fold-increase in red fluorescent AVOs at $48 \mathrm{~h}$ (Fig. 5B). Flow cytometric analysis after acridine orange staining also showed an increase in red fluorescence intensity with sirtinol treatment, indicating an enhancement of AVOs (Fig. 5C). Histogram profiles show the mean fluorescence intensity of the control and drug-treated cells (Fig. 5D). Similar results were observed with MDC staining (Fig. 5E). There was significant autophagic vesicle formation in MCF-7 cells exposed to sirtinol. The morpho- logical characteristics demonstrated that sirtinol induced autophagy in MCF-7 cells.

Inhibition of autophagy sensitizes the sirtinol-induced apoptosis in MCF-7 cells. To explore whether the inhibition of autophagy sensitizes MCF-7 cells to sirtinol-induced apoptotic cell death, we used 3-methyladenine (3-MA), a wellknown inhibitor of autophagy (36). 3-MA blocks autophagic cell death by inhibiting phosphoinositide kinase-3 (PI3K), an enzyme required for autophagy. 3-MA alone had no toxic effect on MCF-7 cells. Following pre-treatment with 3-MA $(0.1 \mathrm{mM})$, sirtinol significantly reduced cell viability in a dose-dependent manner (Fig. 6A). Western blot analysis was performed to assess the expression levels of autophagy- or apoptosis-related protein levels after 3-MA treatment. As shown in Fig. 6B, LC3-II, beclin 1, Atg5 and Atg7 levels were decreased in MCF-7 cells pre-treated with 3-MA compared with those in cells treated with sirtinol alone (Fig. 6B). In addition, 3-MA enhanced sirtinol-induced apoptosis. As shown in Fig. 6C, sirtinol increased the Bax level and decreased the 
A

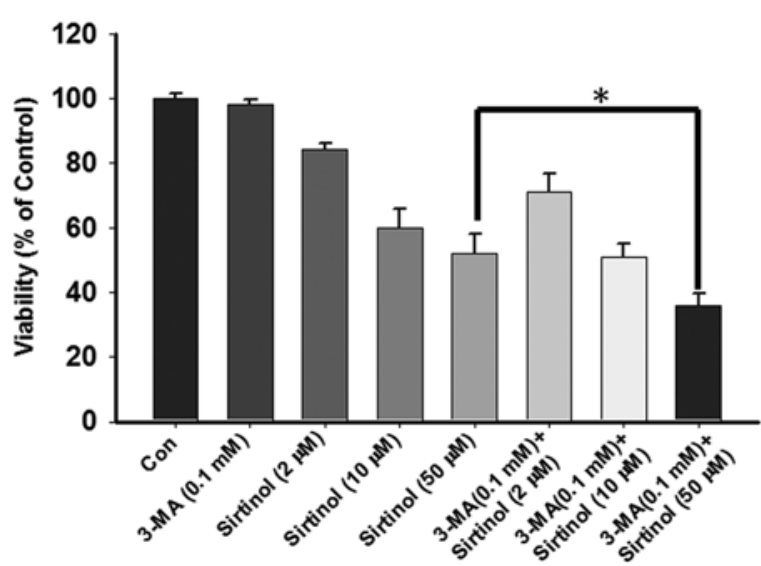

C

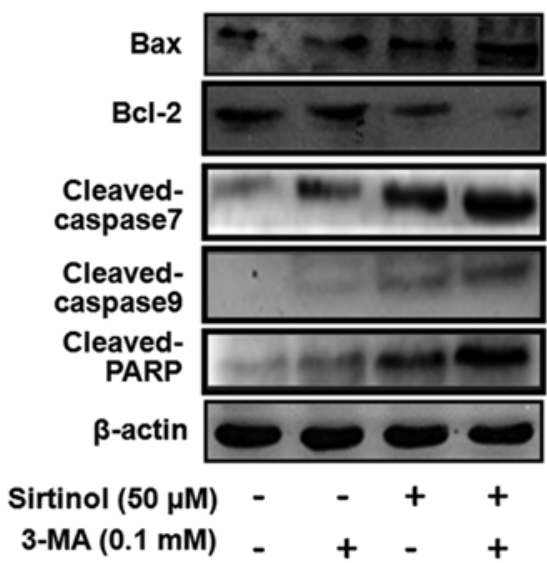

B

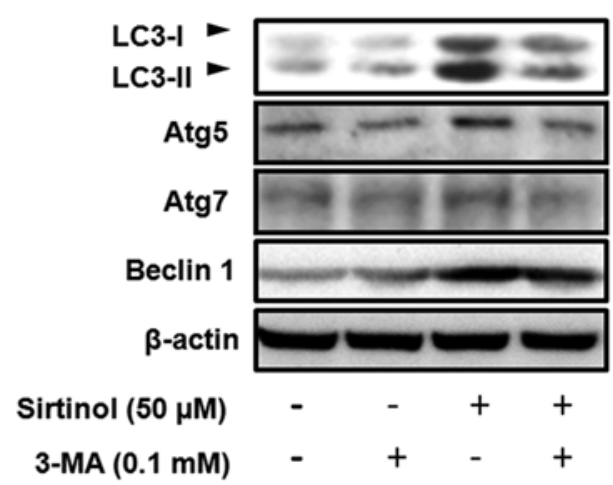

D

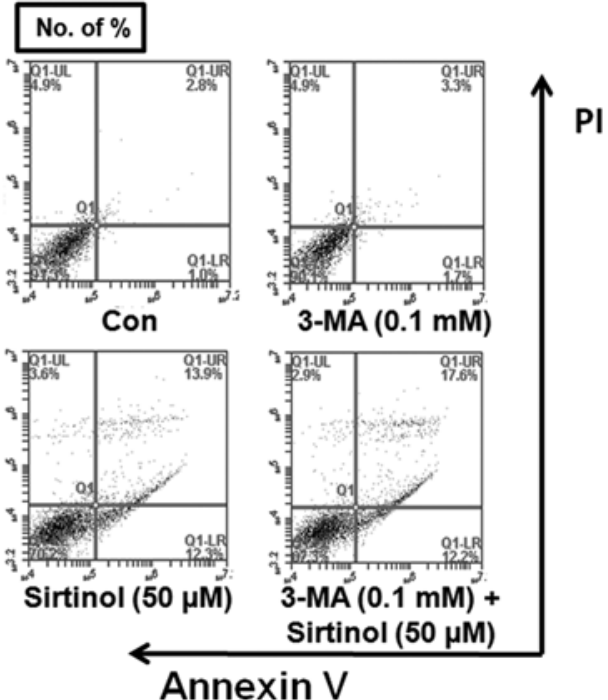

Figure 6. Effect of 3-methyladenine (3-MA) on the sirtinol-induced cell death pathway. (A) MCF-7 cells were pre-treated with 3-MA (0.1 mM) for $6 \mathrm{~h}$ and then with various concentrations of sirtinol for $48 \mathrm{~h}$. The cell viability was measured by MTT assay. (B) Western blot analysis was performed with beclin-1, LC3, Atg5 and Atg7 antibodies. Equal loading and transfer were verified by reprobing the membranes with $\beta$-actin antibody. (C) Western blot analysis was performed with Bax, Bcl-2, cleaved caspase 7, cleaved caspase 9 and cleaved PARP antibodies. Equal loading and transfer were verified by reprobing the membranes with $\beta$-actin antibody. (D) The MCF-7 cells were treated with sirtinol and 3-MA at the indicated concentrations for $48 \mathrm{~h}$. Apoptosis was detected using Annexin V/ propidium iodide (PI)-double staining analyzed by flow cytometry. Con, control.

Bcl-2 level in 3-MA pre-treated MCF-7 cells. The expression levels of caspase 7 and caspase 9 were also altered by sirtinol in the 3-MA-pre-treated MCF-7 cells (Fig. 6C). To confirm that apoptosis was affected by the inhibition of autophagy, cells were subjected to FITC-Annexin V/PI double-staining, followed by flow cytometric analysis to quantify the apoptotic cell populations. Sirtinol significantly increased the number of apoptotic cells among MCF-7 cells pre-treated with 3-MA (Fig. 6D).

\section{Discussion}

SIRTs could be one of the lost links between aging and cancer (37). SIRT1 is the most widely examined member of the SIRT family and is known to modulate cell proliferation, differentiation, apoptosis, as well as migration and invasion $(13,14)$. SIRT1 functions in controlling cellular senescence and specific cancer cell types overexpress SIRT1 $(15,16)$. In this study, we investigated the hypothesis that SIRT is overexpressed in breast cancer cells and that its inhibition would have anticancer effects on human breast cancer. To determine the mechanisms of action by which the SIRT inhibitor, sirtinol, exerts its anticancer effects, we examined its action on apoptotic and autophagic cell death pathways.

First, we measured cell viability and performed cell cycle analysis. We found that sirtinol significantly increased cytotoxicity in a concentration-dependent manner and significantly induced G1 phase arrest in MCF-7 cells. SIRT1/2 expression was significantly reduced by sirtinol treatment, although the effect on SIRT1 expression was more pronounced. Previous studies have demonstrated that class III HDAC inhibitors can also induce senescent-like growth arrest in breast cancer cells (38). SIRT1 has a more prominent role in controlling cell growth and survival as it exists in the same intracellular compartments as most of the cell cycle and death regulators (39). In this study, we used MCF-7 breast cancer cells as they have substantial levels of SIRT1, as well as functional p53, which is a target for acetylation by SIRT1 and SIRT2. The tumor suppressor, p53, can exert anti-proliferative effects, such as growth arrest, apoptosis and cellular senescence, in response to various types of stressors. As expected, sirtinol significantly increased the levels of acetylated p53 in MCF-7 
cells compared with the control culture. Therefore, sirtinol reduced the SIRT1-mediated deacetylation of p53 and increased p53 transcription-dependent cell cycle arrest and apoptosis in MCF-7 cells. These results are similar to those from a previous report indicating that the inhibition of SIRT1 allows the activation of $\mathrm{p} 53$ and BAX gene expression, which induces cell cycle arrest and apoptosis (39).

To explore the mechanism responsible for the anticancer effects of sirtinol, the apoptotic cell death were assessed. Flow cytometric analysis revealed that sirtinol markedly induced apoptosis and subsequently increased the sub-G1 phase cell population. We investigated the exact downstream mechanism of apoptotic cell deaths induced by sirtinol. Sirtinol increased cytochrome $c$ release into the cytoplasm, upregulated the pro-apoptotic protein, Bax, downregulated the anti-apoptotic protein, Bcl-2, and induced PARP cleavage in MCF-7 breast cancer cells. These results were confirmed by Annexin V-FITC assay and DAPI staining. However, while sirtinol potently induced apoptotic cell death in MCF-7 cells, the results concerning autophagy were more significant. Autophagy is becoming an important area of cancer research. Autophagy plays a role in both the promotion and prevention of cancer, and its role may be altered during tumor progression. The inhibition of autophagy may allow the continuous growth of pre-cancerous cells and autophagy can act as a suppressor of cancer (26); then, as a tumor grows, cancer cells may need autophagy to survive nutrient-limiting and low-oxygen conditions, especially in the internal region of the tumor that is poorly vascularized. In addition, autophagy may protect certain cancer cells against ionizing radiation, possibly by removing damaged macromolecules or organelles, such as mitochondria (40). According to our results, the autophagic process caused MCF-7 cell death following sirtinol treatment. These results were confirmed by the sirtinol induction of AVOs in the cytoplasm stained by acridine orange and MDC. Likewise, increases in LC3-II levels and other autophagy-related molecules were observed after sirtinol treatment compared with the control cells and these results correlated closely with their cytotoxic effects.

In truth, whether autophagy promotes cell death or protects cancer cell survival is circumstantial. In this study, sirtinol induced MCF-7 cell death. The inhibition of the early stages of autophagy by the specific inhibitor, 3-MA, resulted in accelerated apoptotic cell death, as revealed by Annexin V/PI staining. Autophagy and apoptosis share many common inducers; however, the current knowledge on the molecular intersections between the autophagic and apoptotic pathways is incomplete and fragmented. It may therefore be necessary to further elucidate the relationship between autophagy and apoptosis following sirtinol treatment in MCF-7 cells. In the present study, we found that sirtinol simultaneously induced p53-mediated apoptosis and caspase-independent autophagy in MCF-7 cells. The inhibition of autophagy by 3-MA-sensitized cells to sirtinol-induced apoptotic cell death, which suggests that the anticancer effects of sirtinol are mainly the result of apoptosis.

Taken together, these results confirm that sirtinol, which decreased the expression of SIRT1 in MCF-7 breast cancer cells, induced cell death effectively, causing cell cycle arrest in the G1 phase and apoptotic cell death, while at the same time inducing autophagic cell death in MCF-7 cells. This evokes hope that a strategy for cancer treatment may be developed based on SIRTs inhibitors.

\section{Acknowledgements}

This study was supported by The Health Fellowship Foundation grants funded by Yuhan Corporation.

\section{References}

1. International Agency for Research on Cancer (IARC): World Cancer Report. http://globocan.iarc.fr/factsheets/populations/ factsheet.asp?uno=900, 2008.

2. Yoo KY, Kang D, Park SK, et al: Epidemiology of breast cancer in Korea: occurrence, high-risk groups, and prevention. J Korean Med Sci 17: 1-6, 2002.

3. Esteve JM and Knecht E: Mechanisms of autophagy and apoptosis: Recent developments in breast cancer cells. World J Biol Chem 26: 232-238, 2011.

4. Cook KL, Shajahan AN and Clarke R: Autophagy and endocrine resistance in breast cancer. Expert Rev Anticancer Ther 11: 1283-1294, 2011.

5. Aguilar H, Solé X, Bonifaci N, et al: Biological reprogramming in acquired resistance to endocrine therapy of breast cancer. Oncogene 29: 6071-6083, 2010.

6. Yue W, Fan P, Wang J, Li Y and Santen RJ: Mechanisms of acquired resistance to endocrine therapy in hormone-dependent breast cancer cells. J Steroid Biochem Mol Biol 106: 102-110, 2007.

7. Notte A, Leclere L and Michiels C: Autophagy as a mediator of chemotherapy-induced cell death in cancer. Biochem Pharmacol 82: 427-434, 2011.

8. Glick D, Barth S and Macleod KF: Autophagy: cellular and molecular mechanisms. J Pathol 221: 3-12, 2010.

9. Minucci S and Pelicci PG: Histone deacetylase inhibitors and the promise of epigenetic (and more) treatments for cancer. Nat Rev Cancer 6: 38-51, 2006.

10. Xu WS, Parmigiani RB and Marks PA: Histone deacetylase inhibitors: molecular mechanisms of action. Oncogene 26: 5541-5552, 2007.

11. Blander G and Guarente L: The Sir2 family of protein deacetylases. Annu Rev Biochem 73: 417-435, 2004.

12. Frye RA: Characterization of five human cDNAs with homology to the yeast SIR2 gene: Sir2-like proteins (sirtuins) metabolize NAD and may have protein ADP-ribosyltransferase activity. Biochem Biophys Res Commun 260: 273-279, 1999.

13. Liszt G, Ford E, Kurtev M and Guarente L: Mouse Sir2 homolog SIRT6 is a nuclear ADP-ribosyltransferase. J Biol Chem 280: 21313-21320, 2005.

14. Liu T, Liu PY and Marshall GM: The critical role of the class III histone deacetylase SIRT1 in cancer. Cancer Res 69: 1702-1705, 2009.

15. Fraga MF and Esteller M: Epigenetics and aging: the targets and the marks. Trends Gene 23: 413-418, 2007.

16. Lim CS: Human SIRT1: a protein biomarker for tumorigenesis? Cell Bio Int 31: 636-637, 2007.

17. Vaquero A, Scher MB, Lee DH, et al: SirT2 is a histone deacetylase with preference for histone H4 Lys 16 during mitosis. Genes Dev 20: 1256-1261, 2006.

18. Jeong J, Juhn K, Lee H, et al: SIRT1 promotes DNA repair activity and deacetylation of Ku70. Exp Mol Med 39: 8-13, 2007.

19. Yeung F, Hoberg JE, Ramsey CS, et al: Modulation of NF- $\kappa \mathrm{B}$-dependent transcription and cell survival by the SIRT1 deacetylase. EMBO J 23: 2369-2380, 2004.

20. Brunet A, Sweeney LB, Sturgill JF, et al: Stress-dependent regulation of FOXO transcription factors by the SIRT1 deacetylase. Science 303: 2011-2015, 2004.

21. Motta MC, Divecha N, Lemieux M, et al: Mammalian SIRT represses forkhead transcription factors. Cell 116: 551-563, 2004.

22. Bouras T, Fu M, Sauve AA, et al: SIRT1 deacetylation and repression of $\mathrm{p} 300$ involves lysine residues 1020/1024 within the cell cycle regulatory domain 1 . J Bio Chem 280: 10264-10276, 2005. 
23. Vaziri H, Dessain SK, Ng Eaton E, et al: Hsir2(SIRT1) functions as an NAD-dependent p53 deacetylase. Cell 107: 149-159, 2001.

24. Bedalov A, Gatbonton T, Irvine WP, Gottschling DE and Simon JA: Identification of a small molecule inhibitor of Sir2p. Proc Natl Acad Sci USA 98: 15113-15118, 2001.

25. Grozinger CM, Chao ED, Blackwell HE, Moazed D and Schreiber SL: Identification of a class of small molecule inhibitors of the sirtuin family of NAD-dependent deacetylases by phenotypic screening. J Biol Chem 276: 38837-38843, 2001.

26. Avalos JL, Bever KM and Wolberger C: Mechanism of sirtuin inhibition by nicotinamide: altering the NAD $(+)$ cosubstrate specificity of a Sir2 enzyme. Mol Cell 17: 855-868, 2005.

27. Bitterman KJ, Anderson RM, Cohen HY, Latorre-Esteves M and Sinclair DA: Inhibition of silencing and accelerated aging by nicotinamide, a putative negative regulator of yeast sir2 and human SIRT1. J Biol Chem 277: 45099-45107, 2002.

28. Ota H, Akishita M, Eto M, Iijima K, Kaneki M and Ouchi Y: Sirt1 modulates premature senescence-like phenotype in human endothelial cells. Mol Cell Cardiol 43: 571-579, 2007.

29. Heltweg B, Gatbonton T, Schuler AD, et al: Antitumor activity of a small-molecule inhibitors of human silent information regulator 2 enzymes. Cancer Res 66: 4368-4377, 2006.

30. Olaharski AJ, Rine J, Marshall BL, et al: The flavoring agent dihydrocoumarin reverses epigenetic silencing and inhibits sirtuin deacetylases. PLoS Genet 1: e77, 2005.

31. Napper AD, Hixon J, McDonagh T, et al: Discovery of indoles as potent and selective inhibitors of the deacetylase SIRT1. J Med Chem 48: 8045-8054, 2005.

32. Saunders LR and Verdin E: Sirtuins: critical regulators at the crossroads between cancer and aging. Oncogene 26: 5489-5504, 2007.
33. Millot C, Millot JM, Morjani H, Desplaces A and Manfait M: Characterization of acidic vesicles in multidrug-resistant and sensitive cancer cells by acridine orange staining and confocal micro-spectrofluorometry. J Histochem Cytochem 45: 1255-1264, 1997.

34. De Duve C, de Barsy T, Poole B, Trouet A, Tulkens P and van Hoof F: Commentary. Lysosomotropic agents. Biochem Pharmacol 23: 2495-2531, 1974.

35. Paglin S, Hollister T, Delohery T, et al: A novel response of cancer cells to radiation involves autophagy and formation of acidic vesicles. Cancer Res 61: 439-444, 2001.

36. Petiot A, Ogier-Denis E, Blommaart EF, Meijer AJ and Codogno P: Distinct classes of phosphatidylinositol 3-kinases are involved in signaling pathways that control macroautophagy in HT-29 cells. J Biol Chem 275: 992-998, 2000.

37. Fraga MF, Agrelo R and Esteller M: Cross-talk between aging and cancer: the epigenetic language. Ann NY Acad Sci 1100: 60-74, 2007.

38. Mahlknecht U and Hoelzer D: Histone acetylation modifiers in the pathogenesis of malignant disease. Mol Med 6: 623-644, 2000.

39. Michishita E, Park JY, Burneskis JM, Barrett JC and Horikawa I: Evolutionarily conserved and nonconserved cellular localizations and functions of human SIRT proteins. Mol Biol Cell 16: 4623-4635, 2005.

40. Arico S, Petiot A, Bauvy C, et al: The tumor suppressor PTEN positively regulates macroautophagy by inhibiting the phosphatidylinositol 3-kinase/protein kinase B pathway. J Biol Chem 276 35243-35246, 2001. 